\title{
Optimize Volume for Planetary Gear Train by using Algorithm Based Artificial Immune System
}

\author{
M.W.Yaw ${ }^{1}$, K.H.Chong ${ }^{2}$ \\ ${ }^{1}$ Department of Electronics and Communication EngineeringUniversitiTenagaNasional (UNITEN) \\ 43009 Kajang, Selangor DarulEhsan, Malaysia, happy_cloud86@hotmail.com \\ ${ }^{2}$ Department of Electronics and Communication Engineering UniversitiTenagaNasional (UNITEN) \\ 43009 Kajang, Selangor DarulEhsan, Malaysia,chongkh@uniten.edu.my
}

\begin{abstract}
Planetary gear train (PGT) had widely used for power transmission in construction equipment and machinery. The main purpose of this paper is to present methods for minimize the volume of PGT as well as the minimum weight for space saving. This paper presents a comparison of develop algorithm based Artificial Immune System (AIS) to find the optimum combination of parameters design for minimize volume of two-stage PGT system. The developed algorithm is hybrid with Genetic Algorithm (GA) based AIS which named as Transform of Artificial Immune System (TRANS-AIS) and Three Parents Cross Transform of Artificial Immune System (X3PAIS). Developed algorithm were tested in three benchmark mathematic test function before apply in the PGT design. The optimum volume from this research are compared with other researchers and it is experimental that the presentation of TRANS-AIS and X3PAIS are as good as (if not superior) to other researchers' algorithm.
\end{abstract}

Key words: Planetary gear train, AIS, GA, optimization.

\section{INTRODUCTION}

PGT have several advantages where they can produce high power density compare to standard parallel axis gear trains, have multiple kinematic combinations, high efficiency, reduction in volume, purely torsional reactions, and coaxial shafting. But there is a main problem faced by designer when design PGT system which is the volume as well as weight of the design combination. Therefore, for the advantages mention above, researcher are focused on research in optimize design of PGT.

KaoutarDaoudi [1] design minimum weight of optimal combination of spur gears by using GA. Takao Yokota [2] described GA in the optimization of gear weight. Abdelmajid [3] optimize gear trains with spur gears based on two level optimizations to reduce the weight. Tianpei Chen [4] optimizes volume of two-stage PGT by using differential evolution algorithm based on mathematical. A. Sri Harsha [5] described optimization of two-stage PGT by using GA to satisfy the strength and geometric constraints. B. Rosic [6] solving multi objective nonlinear optimisation of planetary gear trains and minimizes the weight. Zarefar and Muthukrishnan [7] optimized the weight of helical gear with random search algorithm. FarukMendi [8] presented a dimensional optimization to achieve the optimal dimensions for rolling bearing, gear and gearbox shaft by using GA. Prashant S. Walunj [9] using Finite Element Analysis to minimize vibration by investigates planetary gearbox parameter.

Reduction of volume during optimization gear design, the continuous variables and discrete variables are included in consideration. In traditional method, the optimal design is round to the adjacent discrete points. But this will affect the design point may run out of the specific region. Mostly traditional optimization method is based on gradient algorithm and normally will trap into local minimum search. This leads to incapable in getting favor outcome compared to the modernized Artificial Intelligent (AI) approaches. AI is normally discussed to think or study about patterns or behavior by using computer language [10].

AIS and GA is the two AI methods which have been chosen for simulation in this paper. Operator of GA which is crossover is choose and be composed with AIS with the aim of strengthen the optimization fitness. Proposed algorithms described in this paper are TRANS-AIS and X3PAIS which united the mechanism of improved AIS and GA (using crossover operator). All the information of each algorithm is described in section below. The proposed algorithm will be evaluated with popular benchmark mathematics function. The proposed algorithms were used to optimize the volume of two-stage PGT design.

\subsection{Artificial Immune System}

The idea of AIS is inspirited by the nature biological immune system. Besides that, AIS can be used in solving engineering problem with immunology theory, immune principles, models or function [11]. One of the optimization technique in AIS operation of AIS named as clonal selection algorithm (CSA) was used in this paper. For optimization, the fundamental of CSA in AIS which is the affinity maturation and clonal expansion are used [12]. 
During CSA cloning process, the antigen which can be best recognize by the antibodies will proliferate. Every antigen will have their own specific immune reaction and it will be clone with recreate immune cells in tandem. When the immune cells which can be identify and compete with the specific antigen, this process only consider as success. Plasma and memory cells will be characterized when the newly cloned cells produced. Under mutation process, the plasma cells are subject to create antibody to enrich the genetic variation while the memory cells are in responsible for incursion of antigen in the upcoming immunologic response.

Henceforth in the next mechanism population, selection will depends on which greatest cells have similarity higher with the particular antigen. If mutation process still on going, the high affinity cells with hyper mutation will go into sleep mode while it will undergo withdraw through apoptosis process for the low affinity cells [13]. Theory of CSA process described in Figure 1.

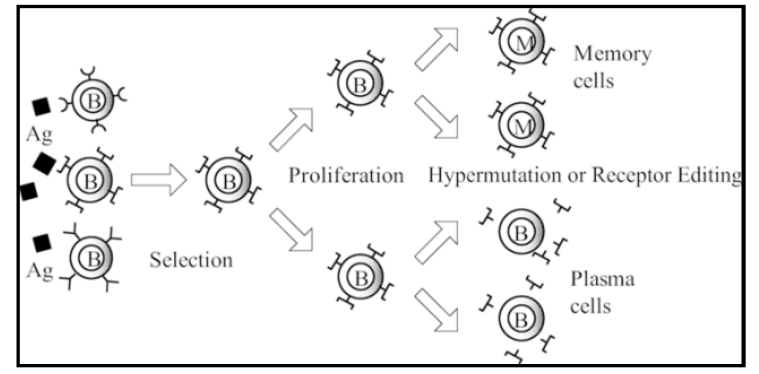

Figure 1: The clonal selection theory

Layout of pseudo code for a typical CSA process is written off as shown in Figure 2.

\begin{tabular}{|l|}
\hline begin AIS \\
$\mathrm{c}:=0$ counter $\}$ \\
Initialize population \\
$\mathrm{Do}:$ \\
Compute affinity \\
Generate clones \\
Mutate clones \\
Replace lowest affinity Ab with a new randomly generated $\mathrm{Ab}$ \\
$\mathrm{c}:=\mathrm{c}+1$ \\
end \\
end AIS
\end{tabular}

Figure 2: Pseudo-code of the CSA

\subsection{Genetic Algorithm}

GA is the members of Evolutionary Algorithm (EA) and it was introduced by John Holland in 1960's [14-16]. GA have since long been used for optimization of high demand in searching for a design with minimal cost for performance of an enquiry [17]. GA is one of the. It used to solve assortment of problems and is proven capable in optimization of determined problem strategy. The crossover operator of GA was chosen to improving AIS algorithm where the idea is comes from the conjugation of bacterial cells.

The purpose of crossover is generates a new set of antibodies by using two or more of members which selected from current population. Crossover process is swapping the genetic criteria between chromosome of same parents or with other parents. There is few type of crossover method in GA but in this paper, the single point crossover method and three parents' crossover method was selected to improve the optimum minimum value.

During single point crossover process, a crossover point will be randomly selected. The new set of chromosome is produced by uniting the first parent of first chromosome until the crossover point with the second parent of chromosome from the point after crossover point until the end of chromosome. The single point crossover process was clearly described in Figure 3.

In Figure 3, the dash line indicates the selected random point of crossover for Antibody A and Antibody B. There are two mature antibody produce after crossover process where the genetics of the chromosome of both parent antibodies was transacted. An enhanced or matured antibody hoped to be born via crossover by expected to inherit the good genetics from two parents.

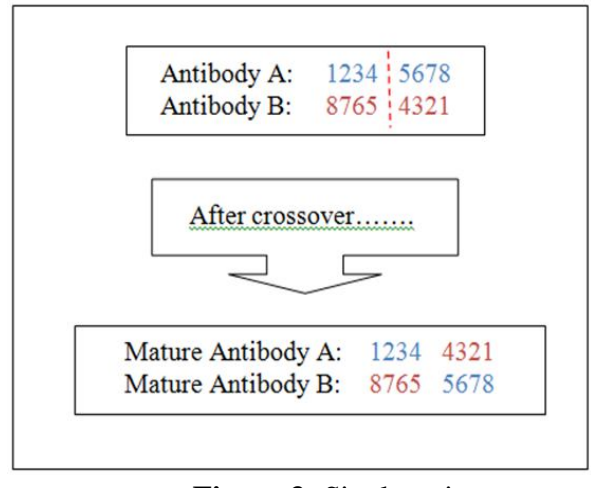

Figure 3: Single-point crossover

Another method of crossover operator was used is the three parents crossover. It means that there are three chromosomes are chosen as parents for crossover process. Every bit of each parent is comparing with each other. In the beginning of crossover process, same point of each bit from first parent and second parent go under comparison. It is used for offspring if the bits are the same; or else the third parent's bit is taking as offspring. Summarized for input and output of the each bit for three parents was represent in Figure 4.

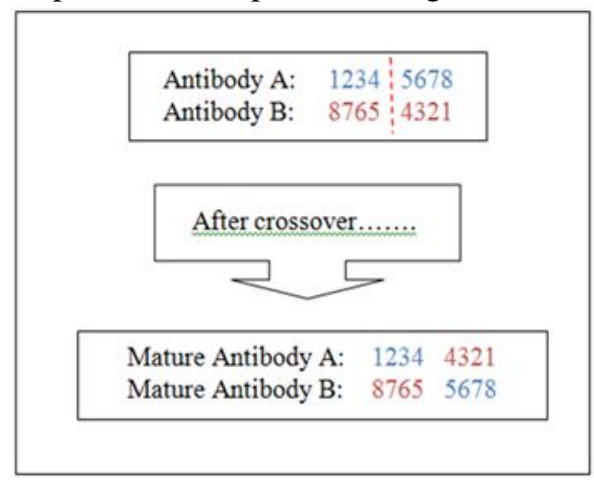

Figure 4: Logic Diagram of input \&output for three Parents crossover 


\subsection{Hybrid of Algorithm}

As mention in previous section, to enhance the optimization of AIS algorithm; conjugate of two genes used in this paper. There are a lot of new reformed bacteria or virus happening from place to place, this brings idea to develop the proposed AI algorithm in this paper. The transformation of new virus can be generated by genetic alteration of a cell and can be lead from the direct uptake or rephrase by external factors of genetic element from the environments. Some species of virus can be transformed naturally or affected by non-natural factors.

\subsubsection{Transform of Artificial Immune System (TRANS-AIS)}

Conjugation genes between two bacterial cells with direct interaction is one of the transformation bacteria processes where it united between two bacterial cells to exchange the genetic material. The crossover process where similar to the idea of conjugation of bacterial cells was applied to improve the performance of AIS algorithm. Cloning process in AIS algorithm is important here to guarantee that the two nominated superior genetics have the greatest antibody. The genetics populations which have better criteria will be undergoing a cloning process.

When the crossover process is finalized, two matured antibodies will produce by swapping the genes of both antibodies. An enhanced and matured antibody had found via crossover which predictable to take over the superior genes. This conjugation procedure is represented by the GA operator, crossover

\subsubsection{Three Parents Cross Transform of Artificial Immune System (X3PAIS)}

The X3PAIS algorithm process was similar to TRANS-AIS. The difference is X3PAIS is conjugated of three bacterial cells and only produce a brand new and mature cell. Direct contact of crossover process is happen in X3PAIS. Since X3PAIS needs to select three superior genetics to undergo crossover process, it's more competitive compare to TRANS-AIS in antibody selection.

When the process of crossover is accomplished, a mature antibody $\mathrm{Y}$ will created by conjugate the genetics of the three selected antibodies. The new established antibody was achieved through crossover process which is probable to become heir of superior genes. This conjugation procedure is represented by the GA operator, crossover.

\section{SIMULATION}

\subsection{Mathematics Test Function}

There are several benchmarks of mathematics test functions with different characteristic used by researchers to do performance checking for each algorithm developed. Researchers will exploit the characteristic of the benchmark mathematics test function for better results. Only three benchmark mathematical test functions used to optimize minimal value by evaluate the developed algorithm in this paper. The test functions are Ackley's, Rosenbrock's and Griewank's function which described as below.

\subsubsection{Ackley's Function}

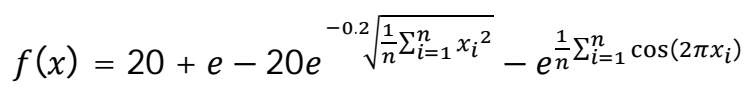

$-32.768 \leq x_{i} \leq 32.768, i=, \ldots \ldots \ldots, n$

global minimum at $x=0$ where $f(x)=0$.

\subsubsection{Rosenbrock's Function}

$f(x)=\sum_{i=1}^{n-1} 100\left(x_{i+1}-x_{i}^{2}\right)^{2}+\left(x_{i}-1\right)^{2}$

$-2.048 \leq x_{i} \leq 2.048, i=1, \ldots \ldots \ldots, n$

global minimum at $x=0$ where $f(x)=0$.

\subsubsection{Griewank's Function}

$f(x)=1+\frac{1}{4000} \sum_{i=1}^{n} x_{i}^{2}-\prod_{i=1}^{n} \cos \left(\frac{x_{i}}{\sqrt{i}}\right)$

$-600 \leq x_{i} \leq 600, i=1, \ldots \ldots \ldots, n$

global minimum at $x=0$ where $f(x)=0$.

\subsection{Planetary Gear Train}

The model of application was a gear box of two-stage PGT which proposed by A. Sri Harsha [5]. The main objective is to minimize the volume of the PGT. Volume for each stage of the gear train which contains sun and planets calculated. Optimize volume for each respective gears equivalent as minimize the volume of the PGT. The objective function is formulated as below.

$V=\frac{\pi}{4}\left[\left(b_{1} *\left({d_{S_{1}}}^{2}+3{d_{P_{1}}}^{2}\right)\right)+\left(b_{2} *\left({d_{S_{2}}}^{2}+3{d_{P_{2}}}^{2}\right)\right)\right]$

where:

$d_{s_{1}}, d_{s_{2}}=$ addendum circle diameter of sun gear.

$d_{p_{1}}, d_{p_{2}}=$ addendum circle diameter of planet gear.

The addendum circle diameter formulated as below.

$d_{a}=(t+2(1-c)) * m$

where:

$\mathrm{t}=$ number of teeth on gear.

$\mathrm{c}=$ addendum modification coefficient of the gear.

$\mathrm{m}=$ module of stages.

The number of teeth on the planetformulated as below.

$t_{p}=\frac{1}{2}(r-2) * t_{s}$

$r=2\left(1+\frac{t_{p}}{t_{s}}\right)$ 
where:

$r=$ reduction ratio of first stage.

To optimize the volume of planetary gear train with proposed algorithm, the design variables of vector $\mathrm{X}$ is created as below:-

$X=\left\{x_{1}, x_{2}, x_{3}, x_{4}, x_{5}, x_{6}, x_{7}, x_{8}, x_{9}\right\}$

$=\left\{t_{1}, m_{1}, b_{1}, c_{1}, r, t_{2}, m_{2}, b_{2}, c_{2}\right\}$

Where:

$\mathrm{t}_{1}=$ number of teeth on sun gear in first stage.

$t_{2}=$ number of teeth in the second stage.

$\mathrm{m}_{1}, \mathrm{~m}_{2}=$ module of first stage and second stage respectively.

$b_{1}, b_{2}=$ width of tooth in first and second stage respectively.

$\mathrm{c}_{1}, \mathrm{c}_{2}=$ modification coefficients of sun gears in first and second stage respectively.

$r=$ reduction of ratio of first stage.

The bounds of the design variable of $\mathrm{X}$ described as Table 1 .

Table 1: Bound for each parameters

\begin{tabular}{|c|c|c|c|c|c|c|c|c|c|}
\hline Variable & $\boldsymbol{t}_{\mathbf{1}}$ & $\boldsymbol{m}_{\mathbf{1}}$ & $\boldsymbol{b}_{\mathbf{1}}$ & $\boldsymbol{c}_{\mathbf{1}}$ & $\boldsymbol{r}$ & $\boldsymbol{t}_{\mathbf{2}}$ & $\boldsymbol{m}_{\mathbf{2}}$ & $\boldsymbol{b}_{\mathbf{2}}$ & $\boldsymbol{c}_{\mathbf{2}}$ \\
\hline $\begin{array}{c}\text { Lower } \\
\text { bound }\end{array}$ & 13 & 5 & 22 & $\frac{4}{17}$ & 5 & 13 & 2 & 110 & $\frac{4}{17}$ \\
\hline $\begin{array}{l}\text { Upper } \\
\text { bound }\end{array}$ & 50 & 9 & 61 & 1 & 10 & 50 & 9 & 140 & 1 \\
\hline
\end{tabular}

\subsubsection{Constraints formulation}

Some constrains have to be set for design the parameters and constant for the PGT system. The model of material chosen is 20CrMnMo. Below is the constant and formulation of constraints.

Table 2: Description of parameters for constraints

\begin{tabular}{|c|l|c|c|}
\hline Parameters & Description & 1st Stage & 2nd Stage \\
\hline$Z_{H}$ & Node region coefficient & 2.22 & 2.25 \\
\hline$Z_{E}$ & Elastic coefficient & 189.98 & 189.98 \\
\hline$Z_{e}$ & Contact ratio coefficient & 0.95 & 0.94 \\
\hline $\mathrm{K}$ & Load factor & 2.89 & 2.95 \\
\hline$Y_{f a}$ & Tooth form factor & 2.29 & 2.32 \\
\hline$Y_{s a}$ & Stress concentration coefficient & 1.73 & 1.73 \\
\hline$Y_{e}$ & Contact ratio coefficient & 1.12 & 1.08 \\
\hline$\sigma_{c}$ & Allowable contact stress & 1033.41 & 1104.47 \\
\hline$\sigma_{b}$ & Allowable bending stress & 499.39 & 521.53 \\
\hline
\end{tabular}

\subsubsection{Contact Stress Calculation}

$\sigma_{1}=Z_{H_{1}} * Z_{E_{1}} * Z_{e_{1}} * \sqrt{\frac{2 * K_{1} * T_{1} *\left(r_{1}+1\right)}{b_{1} * t_{1}^{2} * m_{1}^{2} * r_{1}}} \leq \sigma_{C_{1}}$

$\sigma_{2}=Z_{H_{2}} * Z_{E_{2}} * Z_{e_{2}} * \sqrt{\frac{5.78 * T_{2} *\left(r_{2}+1\right)}{b_{2} * t_{2}{ }^{2} * m_{2}{ }^{2} * r_{2}}} \leq \sigma_{C_{2}}$

where

$\mathrm{T}_{1}, \mathrm{~T}_{2}=$ input torque on the first and second stage.

$r_{1}, r_{2}=$ ratio of tooth number for first and second stage.

\subsubsection{Bending Stress Calculation}

$\sigma_{3}=Y_{F_{a_{1}}} * Y_{S_{a_{1}}} * Y_{e_{1}} * \frac{2 * K_{1} * T_{1}}{b_{1} * t_{1} * m_{1}{ }^{2}} \leq \sigma_{b_{1}}$
$\sigma_{4}=Y_{F_{a_{2}}} * Y_{S_{a_{2}}} * Y_{e_{2}} * \frac{2 * K_{2} * T_{2}}{b_{2} * t_{2} * m_{2}{ }^{2}} \leq \sigma_{b_{2}}$

\subsubsection{Adjacent Condition}

$d_{p_{1}} \leq 2 * a_{12} \sin 60^{\circ}$

$d_{p_{2}} \leq 2 * a_{12}^{\prime} \sin 60^{\circ}$

where

$d_{p_{1}}, d_{p_{2}}=$ addendum circle diameter of the planet gears.

$a_{12}, a_{12}^{\prime}=$ center distance of the gear wheels that are sun and planet gears in first and second stage.

\subsubsection{Tooth Width Condition}

$0.6 * t_{1} \leq \frac{b_{1}}{m_{1}} \leq 1.3 * t_{1}$

$0.6 * t_{2} \leq \frac{b_{2}}{m_{2}} \leq 1.3 * t_{2}$

\section{RESULTS AND DISCUSSION}

\subsection{Mathematics Test Function}

With the aim of validate the presentation of AIS, TRANS-AIS and X3PAIS algorithm, three benchmark mathematic test functions were chosen and the simulations are simulated by using Matlab programming language. The simulation is running under same circumstances for the three mathematic test function as described on last section.

Figure 5-7 described the simulation results of algorithm (AIS, TRANS-AIS and X3PAIS) which were simulated hundreds of iteration. As shown in the results, most of the function values are packed and as close to value zero by using X3PAIS algorithm for each mathematics test function. Comparison of the three mathematics test functions in term of minimum, mean and standard deviation are summarized in Table 3 simulated with the respective algorithm. Therefore, X3PAIS algorithm succeeds to achieve the minimum fitness value compare to others.

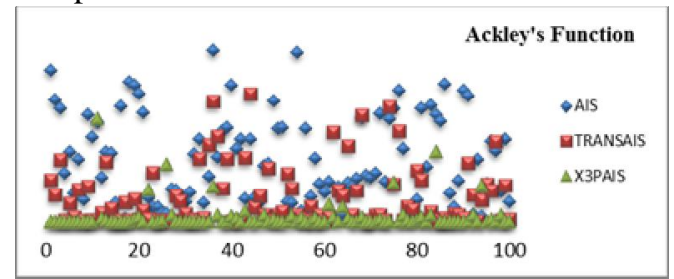

Figure 5: Ackley's Function: hundred experiments data collected

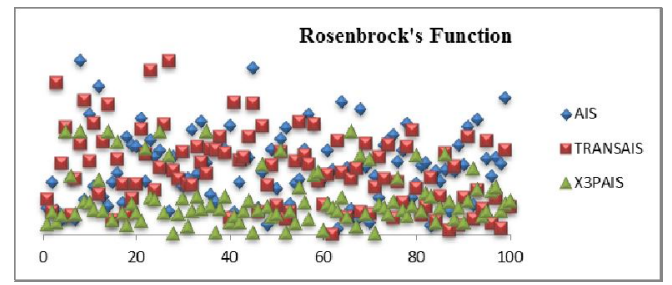

Figure 6: Rosenbrock's Function: hundred experiments data collected 


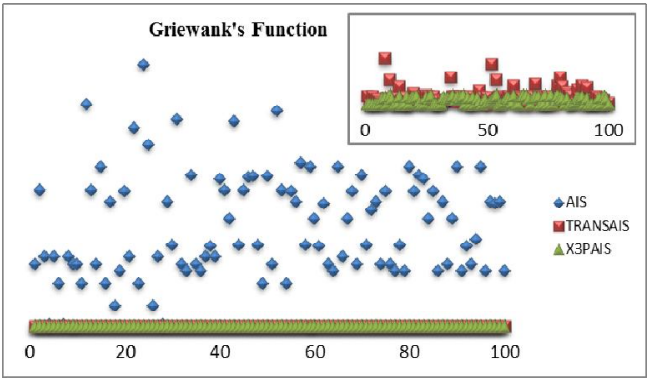

Figure 7: Griewank's Function: hundred experiments data collected

Table 3: Comparisons of Mathematics Functions Results (Minimum, Mean and standard Deviation) for Each Algorithm

\begin{tabular}{|c|c|c|c|c|}
\hline Function & & A & B & C \\
\hline \multirow{4}{*}{ Minimum } & AIS & $3.237714 \mathrm{E}-02$ & $2.903467 \mathrm{E}-03$ & $1.250190 \mathrm{E}-03$ \\
\cline { 2 - 5 } & TRANSAIS & $1.127733 \mathrm{E}-03$ & $2.255263 \mathrm{E}-11$ & $0.000000 \mathrm{E}+00$ \\
\cline { 2 - 5 } Mean & X3PAIS & $9.536773 \mathrm{E}-07$ & $2.255263 \mathrm{E}-11$ & $0.000000 \mathrm{E}+00$ \\
\hline \multirow{3}{*}{$\begin{array}{c}\text { Standard } \\
\text { De viation }\end{array}$} & AIS & $9.862162 \mathrm{E}-01$ & $2.610492 \mathrm{E}-01$ & $1.174186 \mathrm{E}-01$ \\
\cline { 2 - 5 } & TRANSAIS & $3.713335 \mathrm{E}-01$ & $7.421306 \mathrm{E}-07$ & $8.372556 \mathrm{E}-08$ \\
\cline { 2 - 5 } & X3PAIS & $7.812897 \mathrm{E}-02$ & $4.415802 \mathrm{E}-10$ & $4.118649 \mathrm{E}-08$ \\
\cline { 2 - 5 } & AIS & $6.810099 \mathrm{E}-01$ & $2.376589 \mathrm{E}-01$ & $6.035374 \mathrm{E}-02$ \\
\cline { 2 - 5 } & X3PAIS & $2.325998 \mathrm{E}-01$ & $8.837248 \mathrm{E}-10$ & $3.861412 \mathrm{E}-08$ \\
\hline
\end{tabular}

\subsection{Planetary Gear Train}

The minimum volume of the two-stage PGT had calculated and optimized with design of their bound respectively as mention in Table 1.

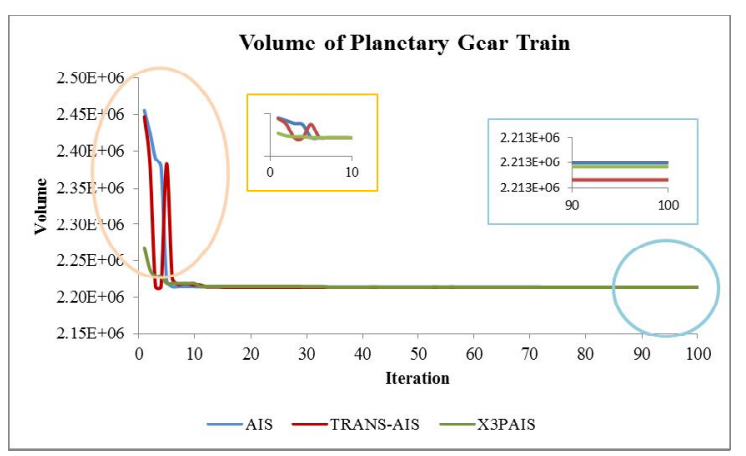

Figure 8: Value of objective function (V) by using each algorithm respectively

Table 4: Comparisons value of volume (V) (minimum, mean and standard deviation) for each algorithm

\begin{tabular}{|l|c|c|c|}
\hline & Minimum & Mean & Standard Deviation \\
\hline AIS & $2.213197 \mathrm{E}+06$ & $2.221514 \mathrm{E}+06$ & $3.946888 \mathrm{E}+04$ \\
\hline TRANS-AIS & $2.213064 \mathrm{E}+06$ & $2.219242 \mathrm{E}+06$ & $3.297880 \mathrm{E}+04$ \\
\hline X3PAIS & $2.213166 \mathrm{E}+06$ & $2.214882 \mathrm{E}+06$ & $6.342265 \mathrm{E}+03$ \\
\hline
\end{tabular}

The comparison of minimum, mean and standard deviation between AIS, TRANS-AIS and X3PAIS were shown in table 4. The results describe that X3PAIS manages to obtain a better results than standard AIS and TRANS-AIS.

Minimum value obtain in simulation were compared with other researchers as described in table 5. A. Sri Harsha [5] using GA, KaoutarDaoudi [18] was using Particle Swarm Optimization (PSO), Differential Evolution (DE) used by
TianPei Chen [4] and Real Coded Genetic Algorithm (RCGA) was introduced by [19] ParidhiRai. The developed algorithm which are TRANS-AIS and X3PAIS in this paper is comparable with other algorithm.

Table 5: Comparisons value of volume,

$\mathrm{V}$ with other researchers

\begin{tabular}{|l|l|}
\hline & Minimum (V) \\
\hline AIS & $2.213197 \mathrm{E}+06$ \\
\hline TRANS-AIS & $2.213064 \mathrm{E}+06$ \\
\hline X3PAIS & $2.213166 \mathrm{E}+06$ \\
\hline GA [5] & $1.204459 \mathrm{E}+07$ \\
\hline PSO [18] & $2.224500 \mathrm{E}+06$ \\
\hline DE [4] & $1.455583 \mathrm{E}+07$ \\
\hline RCGA [19] & $3.844900 \mathrm{E}+05$ \\
\hline
\end{tabular}

\section{CONCLUSION}

The performance of developed algorithm which are TRANS-AIS and X3PAIS algorithm is comparable (if not superior) was clearly described and demonstrate from the simulation in this paper. The developed algorithm was apply into benchmark mathematic test functions and PGT design in minimize of the volume. TRANS-AIS and X3PAIS manage to obtain toward the optimum of best minimum value in benchmark mathematic test function and design of PGT compare to other researchers. Performance improvements of the TRANS-AIS and X3PAIS algorithm still can be done by tweaking the selection criteria and parameters in future research.

\section{REFERENCES}

1. K. Daoudi and E. M. Boudi. Genetic Algorithm Approach for Spur Gears Design Optimization, 2018 International Conference on Electronics, Control, Optimization and Computer Science (ICECOCS), pp. 1-5, 2018. https://doi.org/10.1109/ICECOCS.2018.8610520

2. T. Yokota, T. Taguchi and M. Gen. A solution method for optimal weight design problem of the gear using genetic algorithms, Journal of Computers \& Industrial Engineering, vol. 35, pp.523-526, 1998.

3. A. A. Taleb, M. El Amine and Mohd. Sallaou. Multi-level Optimization of Gear Trains with Spur Gears,2019 5th International Conference on Optimization and Applications (ICOA), pp.1-4, 2019. https://doi.org/10.1109/ICOA.2019.8727684

4. T.P. Chen, Z.Y. Zhang, D.F. Chen and Y.Z. Li.The Optimization of Two-Stage Planetary Gear Train Based on Mathmatica. Pervasive Computing and the Networked World, pp.122-136, 2013.

5. A. Sri Harsha and K. MallikarjunaRao. The Optimization of Two-Stage Planetary Gear Train Based on Genetic Algorithm. International Journal \& Magazine Engineering, Techonology, Management and Research, vol. 4, pp.525-528, 2017.

6. B.Rosic, S.Radenovic L.J. Jankovic and M. Milojevic. Optimization of Planetary Gear Train Using 
Multiobjective Genetic Algorithm, Journal of the Balkan Tribological Association, vol. 17, pp. 462-475, 2011.

7. H. Zarefar and S. N. Muthukrishnan. Computer-aided optimal design via modified adaptive random-search algorithm, Computer-Aided Design, vol. 25, pp.240-248, 1993.

8. F. Mendi, T. Başkal, K. Boran and F.E. Boran. Optimization of module, shaft diameter and rolling bearing for spur gear through genetic algorithm, Expert Systems with Applications, vol. 37, pp.8058-8064, 2010.

https://doi.org/10.1016/j.eswa.2010.05.082

9. Prashant S. Walunj, V. N. Chouguleand Anirban C. Mitra. Investigation of Modal Parameters on Planetary Gearbox using Finite Element Analysis to Minimize Vibration, International Journal of Analytical, Experimental and Finite Element Analysis, vol. 2, pp.147-152, 2015.

10. Abdulnaser S. H. Al-MSloum. The Role of Artificial Intelligence in Supporting the Supply Chain, According to the Kingdom's Vision 2030, International Journal of Advanced Trends in Computer Science and Engineering, vol. 9, pp.1050-1055, 2020. https://doi.org/10.30534/ijatcse/2020/23922020

11. L. N. De Castro, J. Timmis.Artificial immune systems: A new computational approach, New York, Springer-Verlag, 2002.

12. De Castro and Von Zuben F. J. Learning and Optimization using Clonal Selection Principle, IEEE on Evolutionary Computation, Special Issue on Artificial Immune system, vol. 6, pp. 239-251, 2002.

13. T. B. Kepler and A. S. Perelson. Somatic Hypermutation in B Cells: An Optimal Control Treatment, Journal of Theoretical Biology,vol. 164, pp. 37-64, 1993.

https://doi.org/10.1006/jtbi.1993.1139

14. J. H. Holland. Adaption in Natural and Artificial Systems: An introductory Analysis with Application to Biology, Control and Artificial Intelligence, United States of America: MIT Press, 1992.

15. R. L. Haupt. Introduction to Optimization. Practical Genetic Algorithms,PP.1-25, 2004.

16. I. A. Korejo and S.X. Yang. A Comparative Study of Adaptive Mutation Operators for Genetic Algorithms,Inproc of VIII Metaheuristics International Conf, pp.1-10, 2009.

17. Swati V. Chande. Database Query Optimization using Genetic Algorithms: A Systematic Literature Review, International Journal of Advanced Trends in Computer Science and Engineering, vol. 8, pp.1903-1913, 2019. https://doi.org/10.30534/ijatcse/2019/14852019

18. K. Daoudi and E. M. Boudi. Optimal Volume Design of Planetary Gear Train Using Particle Swarm Optimization,4th International Conf on Optimization and Applications (ICOA), PP.1-4, 2018.

19. Paridhi Rai, Aman Agrawal, Mohan L. Saini, Chinmay Jodder and Asim Gopal Barman.Volume Optimization of Helical Gear with Profile Shift Using Real Coded Genetic Algorithm, International Conference on
Robotics and Smart Manufacturing, vol.133, pp.718-724, 2018.

https://doi.org/10.1016/j.procs.2018.07.127 